\title{
On Operators and Elementary Functions in Clifford Analysis
}

\author{
W. Sprössig
}

Dedicated to Prof. L. von Wolfersdorf on the occasion of his 65th birthday

\begin{abstract}
In this paper a survey on the construction of Clifford regular elementary functions by Fueter's mapping is given. Furthermore, using a suitable decomposition of the Dirac operator an application of the $\bar{\partial}$-problem is lined out.
\end{abstract}

Keywords: Clifford regular elementary functions, Fueter's method, $\bar{\partial}$-problem

AMS subject classiflcation: $30 \mathrm{G} 35$

\section{Indroduction}

The effective application of methods of Clifford analysis to partial differential equations needs systems of elementary functions which are Clifford regular and still satisfy most of the properties which we know from the complex plane. The reason for writing this paper is to show presumed users of Clifford-valued elementary functions the considerable similarity to classical complex elementary functions and to reduce the shyness in applying higher-dimensional elementary functions. In this paper we will give a review on Fueter's method and will construct some important Clifford regular elementary functions which knowledge seems to be useful in transform analysis.

We will mention here important authors which deliver contributions in this field. In his early papers [5 - 7] R. Fueter, a follower of D. Hilbert, formulated a method to transfer complex analytic functions to Clifford regular ones. Later M. Sce [18], A. Sudbury [22], M. Imaeda [9], F. Sommen [20, 21], P. Lounesto and P. Bergh [15], G. Jank and F. Sommen [11], H. Leutwiler [12 - 14], M. Marinov [16] and K. Nono [17] made attempts in generalizing classical elementary functions in a hypercomplex sense. Further, the reader can find contributions to this subject in $[1,2,4]$.

Using the power series expansion of an exponential function with a paravector argument we deduce a "full" class of so-called radially regular elementary functions including a paravector-valued logarithm. Most of the expected properties could be maintained. We continue with a suitable decomposition of the Dirac operator and study the operators which there occur. In this part we use results of J. de Graaf [3] and N. van Acker [23]. On the basis of papers by M. Sce [19] and T. Qian [18] we are able to describe

W. Sprössig: Univ. of Mining \& Techn., Dept. Math. \& Comp. Sci., D-09695 Freiberg 
at least in the case of quaternions the transform of radially regular function to quaternionic regular functions. Finally, we apply these results for the construction of kernel functions of the $\bar{\partial}$-problem.

\section{Preliminaries}

Let $\mathbb{R}^{0, n}$ be the anti-Euclidean space with the basis $\left\{e_{1}, \ldots, e_{n}\right\}$ and the quadratic form $Q(x)=-\sum_{i=1}^{n} x_{i}^{2}$. We consider the $2^{n}$-dimensional real Clifford algebra $C \ell_{0, n}$ which is generated by $\left\{e_{1}, \ldots, e_{n}\right\}$ and contains copies of $\mathbb{R}$ and $\mathbb{R}^{0, n}$. The multiplication rule is defined by $e_{i} e_{j}+e_{j} e_{i}=-2 \delta_{i j}$. In $C \ell_{0, n}$ a basis is given by $e_{0}, e_{1}, \ldots, e_{n}, e_{1} e_{2}, \ldots, e_{n-1} e_{n}$, $\ldots, e_{1} \cdots e_{n}$. Elements of the type $x=x_{1} \ldots k e_{\ell_{1}} \cdots e_{\ell_{k}} \quad\left(1 \leq \ell_{1}<\ldots<\ell_{k} \leq n\right)$ are called $k$-vectors.

For $k$-vektors the conjugation is defined by $\bar{x}=(-1)^{\frac{k(k+1)}{2}} x$. Elements $x=x_{0}+\underline{x}$ with $\underline{x}=\sum_{i=1}^{n} x_{i} e_{i}$ are called paravectors. Furthermore, $x_{0}$ is called scalar part, $x_{0}:=$ Sc $x, \underline{x}$ is called vector part, $\underline{x}:=\operatorname{Vec} x$, and $x-x_{0}$ is called imaginary part, $x-x_{0}:=\operatorname{Im} x$. Obviously, $x \bar{x}=\sum_{i=1}^{n} x_{i}^{2}$ for $x=x_{0}+\underline{x}$. Denote $\omega(x):=\underline{x} /|\underline{x}| \in S^{n}$, where $S^{n}$ is the unit sphere in $\mathbb{R}^{n}$.

\section{Paravector-valued elementary functions}

Let $x$ be a paravector in $C \ell_{0, n}$. The following statement can be easily shown.

Proposition 3.1. For an arbitrary $\varepsilon>0$ it is always possible to find a sufficiently large number $N$ such that for any $r, s>N$

$$
\left|\sum_{k=r}^{s} \frac{x^{k}}{k !}\right| \leq \sum_{k=r}^{s} \frac{K^{k}|x|^{k}}{k !}<\varepsilon
$$

holds where $K$ is a constant which only depends on $n$ and satisfies the inequality $|x y|<$ $K(n)|x||y|$.

Inequality (3.1) gives us the possibility to define elementary functions similarly to the case of one complex variable. First of all we have to introduce an exponential function:

Definition 3.2. For a paravector $x \in C \ell_{0, n}$ the exponential function $e^{x}$ is defined by $e^{x}=\sum_{k=0}^{\infty} \frac{x^{k}}{k !}$.

Similarly to the complex case one can prove the following properties.

Theorem 3.3. Let $x$ be a paravector in $C \ell_{0, n}$. Then we have:

(i) $e^{x}=e^{x_{0}}(\cos |\underline{x}|+\omega(x) \sin |\underline{x}|)$.

(ii) $e^{x}=\lim _{m \rightarrow \infty}\left(1+\frac{x}{m}\right)^{m}$.

(iii) $e^{x+y}=e^{x} e^{y}$ if $x y=y x$. 
Proof. We will only prove property (ii). Because of $\left(\frac{1}{k !}-\frac{\left(m_{k}^{m}\right)}{m^{k}}\right)>0$ we obtain

$$
\left|e^{x}-\left(1+\frac{x}{m}\right)^{m}\right| \leq \sum_{k=0}^{\infty}\left(\frac{1}{k !}-\frac{\left(\begin{array}{c}
m \\
k
\end{array}\right)}{m^{k}}\right)(K|x|)^{k}=e^{|K x|}-\left(1+\frac{|K x|}{m}\right)^{m} .
$$

For $m \rightarrow \infty$ this difference tends to zero

Corollary 3.4. Furthermore, we have:

(i) $e^{x} \neq 0$.

(ii) $\left|e^{x}\right|=e^{x_{0}}$.

(iii) $e^{-x} e^{x}=1$

(iv) $e^{k \underline{x}}=\left(e^{\underline{x}}\right)^{k} \quad$ (Moivre's Formula)

(v) $e^{\omega(x) \pi}=-1$.

Proof. This is a straightforward consequence of Theorem 3.3

Let $x$ be a paravector in $C \ell_{0, k}$. Then hyperbolic and trigonometric functions are defined by

$$
\sinh x=\frac{e^{x}-e^{-x}}{2} \quad \text { and } \quad \cosh x=\frac{e^{x}+e^{-x}}{2}
$$

and, for $|\underline{x}| \neq 0$,

$$
\sin x=\frac{e^{x \omega(x)}-e^{-x \omega(x)}}{2} \omega(x) \quad \text { and } \quad \cos x=\frac{e^{x \omega(x)}+e^{-x \omega(x)}}{2} .
$$

Corollary 3.5. Immediately the representations

$$
\begin{aligned}
\cosh x & =\cos h x_{0} \cos |\underline{x}|+\omega(x) \sin h x_{0} \sin |\underline{x}| \\
\sinh x & =\sin h x_{0} \cos |\underline{x}|+\omega(x) \cos h x_{0} \sin |\underline{x}| \\
\cos x & =\cos x_{0} \cos h|\underline{x}|+\omega(x) \sin h|\underline{x}| \sin x_{0} \\
\sin x & =\sin x_{0} \cos h|\underline{x}|+\omega(x) \sin h|\underline{x}| \cos x_{0}
\end{aligned}
$$

follow. The right-hand sides of $\sin x$ and $\cos x$ can be used by definition in the case of $|\underline{x}|=0$.

Proof. For the proof we refer to our book [8: pp. $53-55]$

Definition 3.6. Let $x$ be a paravector in $C \ell_{0, n}$ with $x \neq x_{0}<0$. Then $\log x$ is defined by

$$
\log x=\ln |x|+\omega(x) \arccos \frac{x_{0}}{|x|} \quad\left(|\underline{x}| \neq 0 \text { or }|\underline{x}|=0, x_{0}>0\right) .
$$

Theorem 3.7. Let $x$ be a paravector in $C \ell_{0, n}$ with $x \neq x_{0}<0$. Then we have:

(i) $e^{\log x}=x$ and $\log e^{x}=x$.

(ii) $\log 1=0$ and $\log e_{i}=\frac{\pi}{2} \quad(i=1, \ldots, n)$.

(iii) $1-\frac{1}{|x|}-\arctan \frac{|\underline{x}|}{\left|x_{0}\right|} \leq \log |x| \leq|x|-1+\arctan \frac{|\underline{x}|}{\left|x_{0}\right|}$.

(iv) $\log (x y)=\log x+\log y$ if $x y=y x$.

Proof. The proof follows immediately from Definition 3.6 
Finally, it is also possible to introduce a general power function.

Definition 3.8. Let $\alpha$ be a real number. The general power function $x^{\alpha}$ is defined by $x^{\alpha}=e^{\alpha \log x}$.

The following example will confirm this definition.

Exámple 3.9. Let $x=\underline{x}$ and $\alpha=\frac{1}{n}$. We obtain after a straightforward calculation

$$
\underline{x}^{\frac{1}{n}}=\sqrt[n]{|\underline{x}|}\left[\cos \left(\frac{\pi}{2 n}+\frac{2 k i}{n}\right)+\omega(x) \sin \left(\frac{\pi}{2 n}+\frac{2 k \pi}{n}\right)\right]
$$

for $k=0,1, \ldots, n-1$.

\section{On the Cauchy-Fueter operator}

In order to prepare differentiability properties of the elementary functions introduced above we need a suitable decomposition of the so-called Cauchy-Fueter operator

$$
\partial=\partial_{0}+D \quad \text { where } \partial_{0}=\frac{\partial}{\partial x_{0}} \text { and } D=\sum_{i=1}^{n} e_{i} \partial_{i}, \partial_{i}=\frac{\partial}{\partial x_{i}}
$$

With the denotations

$$
L=\sum_{i=1}^{n} e_{i} L_{i}(x) \text { with } L_{i}(x)=|\underline{x}| \partial_{i}-x_{i} \dot{\ell}_{\omega} \quad \text { and } \quad \ell_{\omega}=\sum_{i=1}^{n} \omega_{i} \partial_{i}
$$

we will obtain the decomposition

$$
\partial=\frac{1}{2}\left(\partial_{0}-\frac{1}{|\underline{x}|} L+\omega \ell_{\omega}\right)
$$

For a better understanding it is useful to deduce properties of the decomposition operators $L$ and $\ell_{\omega}$.

Proposition 4.1. Let $f \in C^{1}\left(\mathbb{R}^{n}\right)$ be a paravector-valued function. Then:

(i) $l_{\omega} \underline{x}=\omega$.

(ii) $\ell_{\omega} \omega=0$.

(iii) $\ell_{\omega} f=\frac{d}{d|\underline{x}|} f$.

(iv) $|\underline{x}| \partial_{j} \omega_{k}=\delta_{j k}-\omega_{k} \omega_{j}=L, \omega_{k}$.

(v) $\sum_{i=1}^{n} \omega_{i} L_{i}=\operatorname{Sc} \omega L=0$.

Proof. These relations are simple consequences from the definition of the operators $L_{i}$ and $\ell_{w}$ 


\section{Proposition 4.2.}

(i) Let $f \in C^{1}(\mathbb{R})$ and $f=f(|\underline{x}|)$ a paravector-valued function. Then $L f=0$.

(ii) Let $\varphi \in C^{1}(\mathbb{R})$ and $\varphi=\varphi(\omega)$ a scalar-valued function. Then

$$
L \varphi=|\underline{x}|\left[\sum_{k=1}^{n} \partial_{\omega_{k}} \varphi e_{k}-\sum_{k=1}^{n} \omega_{k} \partial_{\omega_{k}} \varphi\right]=|\underline{x}|\left[\operatorname{grad}_{\omega} \varphi-\omega\left(\operatorname{grad}_{\omega} \varphi \cdot \omega\right)\right] .
$$

(iii) $\ell_{\omega} \varphi(\omega)=0$ is valid.

Proof. Statement (i): For $j=1, \ldots, n$ we obtain by definition $L_{j}(|\underline{x}|)=|\underline{x}| \partial_{j}|\underline{x}|-$ $x_{j} \ell_{\omega}|\underline{x}|=0$. Furthermore, we have

$$
L_{j} f(|\underline{x}|)=|\underline{x}| \partial_{j} f-x_{j} \sum_{i=1}^{n} \omega_{i} \partial_{i} f=\ell_{\omega} f\left(|\underline{x}| \partial_{j}|\underline{x}|-x_{j} \ell_{\omega}|\underline{x}|\right)=\ell_{\omega} f L_{j}|\underline{x}|=0 .
$$

Statement (ii): Let $\varphi=\varphi(\omega)$. Then

$$
L \varphi=|\underline{x}| \operatorname{grad}_{\omega} \varphi-\omega\left[\left(\operatorname{grad}_{\omega} \varphi\right) \cdot \omega\right]
$$

where $\operatorname{grad}_{\omega} \varphi=\sum_{i=1}^{n} e_{i} \partial_{\omega_{i}}=\varphi$. Indeed, we have

$$
(L \varphi)(\omega)=\sum_{i=1}^{n} e_{i} L_{i} \varphi(\omega)=|\underline{x}| \sum_{i=1}^{n}\left|e_{i} \partial_{i}-\omega_{i} \ell_{\omega}\right| \varphi(\omega) .
$$

Setting now $\varphi^{*}(x):=. \varphi(\omega)$

$$
\left(\partial_{j} \varphi^{*}\right)(x)=\sum_{j=1}^{n} \partial_{\omega_{k}} \varphi \partial_{j} \omega_{k}=\sum_{k=1}^{n} \partial_{\omega_{k}} \varphi\left(\delta_{j k}-\omega_{j} \omega_{k}\right) .
$$

Hence,

$$
\begin{aligned}
\sum_{j=1}^{n} e_{j} \partial_{j} \varphi^{*}(x) & =\sum_{j=1}^{n} \sum_{k=1}^{n} e_{j} \partial_{\omega_{k}} \varphi \partial_{j} \omega_{k} \\
& =\sum_{k=1}^{n} \sum_{j=1}^{n} e_{j} \partial_{\omega_{k}} \varphi \delta_{j k}-\sum_{k=1}^{n} \sum_{j=1}^{n} e_{j} \omega_{j} \partial_{\omega_{k}} \varphi \omega_{k} \\
& =\operatorname{grad}_{\omega} \varphi-\omega \sum_{k=1}^{n} \partial_{\omega_{k}} \varphi \omega_{k} \\
& =\operatorname{grad}_{\omega} \varphi_{1}-\omega\left(\operatorname{grad}_{\omega} \varphi \cdot \omega\right)
\end{aligned}
$$

Statement(iii): We have

$$
\ell_{\omega} \varphi(\omega)=\sum_{k=1}^{n} \partial_{k} \varphi \ell_{\omega} \omega_{k}=\operatorname{grad}_{\omega} \varphi \cdot \ell_{\omega} \omega=0
$$

Thus the proposition is proved 
Remark. Following [3] we get for $L$ the vector representation

$$
L=\sum_{j=1}^{n} e_{j}\left(v^{j} \cdot D\right)
$$

where $v^{j}=|\underline{x}| e_{j}-\omega_{j} \underline{x}$, which can be easily seen by the help of Proposition 3.1

Proposition 4.4. $\omega L$ is the spherical Dirac operator.

Proof. Because of Proposition 4.1/(iv) we find

$$
\begin{aligned}
\omega L & =\sum_{j \neq k} e_{j} e_{k} \omega_{j} L_{k} \\
& =\sum_{j<k} e_{j} e_{k}\left(\omega_{j} L_{k}-\omega_{k} L_{j}\right) \\
& =\sum_{j<k} e_{j} e_{k}\left[\left(\omega_{j}|\underline{x}| \partial_{k}-\omega_{j} x_{k} \ell_{\omega}\right)-\omega_{k}|\underline{x}| \partial_{j}-x_{j} \omega_{k} \ell_{\omega}\right] \\
& =\sum_{j<k} e_{j} e_{k}\left(x ; \partial_{k}-x_{k} \partial_{j}\right)
\end{aligned}
$$

and the statement is proved

Theorem 4.5. We have the equality

$$
(\omega L+L \omega) u=(1-n) u
$$

for the anti-commutator. Special cases.

(i) If $u \equiv u_{0}(|\underline{x}|)$, where $u_{0}$ a real-valued function, then $L \omega u_{0}=(1-n) u_{0}$.

(ii) If $u=\omega$, then $(\omega L) \omega=(n-1) \omega$.

Proof. We have

$$
L \omega=\sum_{j, k=1}^{n} e_{j} e_{k} L_{j} \omega_{k}+\sum_{j, k=1}^{n} e_{j} e_{k} \omega_{k} L_{j} .
$$

Using Proposition 4.1/(iii)-(iv) we get

$$
L \omega=\sum_{j, k=1}^{n}\left(\delta_{j k}-\omega_{k} \omega_{j}\right) e_{j} e_{k}-\omega L=-n+1-\omega L
$$

and the statement is proved

Proposition 4.6. The Cauchy-Fueter operator permits the decomposition

$$
\partial=\frac{1}{2}\left(\partial_{0}+\omega \ell_{\omega}\right)-\frac{1}{2} \frac{1}{|\underline{x}|} L .
$$

Proof. It is easy to see that

$$
\omega L=\omega \sum_{j=1}^{n} e_{j}\left[|\underline{x}| \partial_{j}-x_{j} \ell_{\omega}\right]=\underline{x} D+|\underline{x}| \ell_{\omega} .
$$

From this we obtain the assumption by a straightforward calculation 
Corollary 4.7. Let $\xi=\underline{\xi}(|\underline{x}|)$. Then:

(i) $L(\xi \omega)=(n-3) \xi+2(\xi, \omega) \omega$.

(ii) $D(\xi \omega)=\frac{1}{|\underline{x}|} L(\xi \omega)+\frac{d}{d|\underline{x}|} \xi$. deliver

Proof. Corollary 4.6, the generalized Leibniz rule [8: p. 40] and Proposition 4.4

$$
D(\xi \omega)=(D \xi) \omega-\xi D \omega-2 \sum_{j=1}^{n} \xi_{j} \partial_{j} \omega=\omega \xi^{\prime} \omega-2 \sum_{j=1}^{n} \sum_{k=1}^{n} e_{k} \xi\left(\partial_{j} \omega_{k}\right)+\frac{n-1}{|\underline{x}|} \xi
$$

Furthermore, it follows

$$
\begin{aligned}
D(\xi \omega) & \left.=\omega\left(\frac{d}{d|\underline{x}|} \xi\right) \omega+\frac{(n-1)}{|\underline{x}|} \xi-\frac{2}{|\underline{x}|} \sum_{j=1}^{n} \sum_{k=1}^{n} e_{k} \xi_{j} \mid \delta_{j k}-\omega_{j} \omega_{k}\right] \\
& =\omega\left(\frac{d}{d|\underline{x}|} \xi\right) \omega+\frac{n-1}{|\underline{x}|} \xi-\frac{2}{|\underline{x}|} \xi+\frac{2}{|\underline{x}|}(\xi, \omega) \omega \\
& =\omega\left(\frac{d}{d|\underline{x}|} \xi\right) \omega+\frac{1}{|\underline{x}|}(n-3) \xi+\frac{2(\xi, \omega)}{|\underline{x}|} \omega .
\end{aligned}
$$

Obviously, we get from $\ell_{\omega}(\xi \omega)=\left(\frac{d}{d|\underline{x}|} \xi\right) \omega$ and Corollary 4.6

$$
L(\xi \omega)=-|\underline{x}|\left(D(\xi \omega)-\omega\left(\frac{d}{d|\underline{x}|} \xi\right) \omega\right)=(n-3) \xi+2(\xi, \omega) \omega
$$

and the proof is finished

\section{Fueter's mapping}

The elementary functions introduced above are not Clifford regular. Above all this is caused by the occurence of the operator $L$ and his action

$$
L\left(u_{0} \omega\right)=(1-n) u_{0}
$$

(cf. Theorem 4.5). Such a cross-mapping which acts from the vector part to the real part is disturbing the structure of this simple type of elementary functions. In order to maintain such differentiability properties it seems to be useful to introduce the "reduced" operator $\partial_{r a}=\frac{1}{2}\left(\partial_{0}-\omega \ell_{\omega}\right)$. Analogously to the complex case we abbreviate $\partial_{r a} u=: u^{\prime}$.

Definition 5.1. Let

$$
f=f_{0}+\omega(x) f_{1}, \quad f_{i}: \mathbb{R}^{n} \oplus \mathbb{R}^{1} \rightarrow \mathbb{R}^{1}, f_{i}=f_{i}\left(x_{0},|\underline{x}|\right)(i=1,2), h=h_{0} \omega(x)|\underline{h}| .
$$

Such a paravector-valued function $f$ is called radially differentiable or radially Clifford regular if

$$
\lim _{h \rightarrow 0} \frac{[f(x+h)-f(x)] \bar{h}}{|h|^{2}}=A_{f}(x)
$$


exists.

Corollary 5.2. For radially differentiable functions $A_{f}(x)=f^{\prime}(x)$ holds.

Corollary 5.3. The paravector-valued function $f=f_{0}+\omega f_{1}$ is radially differentiable if and only if $\bar{\partial}_{\text {ra }} f=0$

Corollary 5.4. All above defined elementary functions are radially Clifford regular. Hence it follows:

(i) $\left(e^{x}\right)^{\prime}=e^{x}$

(ii) $(\sin x)^{\prime}=\cos x$ and $(\cos x)^{\prime}=-\sin x$.

(iii) $(\sinh x)^{\prime}=\cosh x$ and $(\cosh x)^{\prime}=\sinh x$.

(iv) $(\log x)^{\prime}=\frac{1}{x}$.

(v) $x^{\alpha}=\alpha x^{\alpha-1} \quad(\alpha \in \mathbb{R})$.

Proof. The proof follows straightforward by using the elementary relations $\ell_{\omega} \omega=$ $0, \ell_{\omega}|x|=\frac{|\underline{x}|}{|x|}$ and $\varepsilon=\ell_{\omega} \underline{x}=\omega$

Now we will demonstrate how to transform these radially differentiable clementary functions to Clifford regular functions. The key-idea goes back to $\mathrm{R}$. Fueter and was later generalized by M. Sce [19], F. Sommen [20, 21] and T. Qian [18]. Let $u=u\left(x_{0},|\underline{x}|\right)$ and $v=v\left(x_{0},|\underline{x}|\right)$ real-valued functions and $h=u+\omega v$. We will denote by $\tau_{n}$ the mapping

$$
q=\tau_{n}(h)=\kappa_{n} \Delta^{\frac{(n-1)}{2}} h,
$$

where $\Delta$ denotes the Laplacian and $\kappa_{n}$ a normalization factor. For even $n$ the operator $\Delta^{\frac{n-1}{2}}$ has to be considered as Fourier multiplier operator induced by the symbol $(2 \pi i|\xi|)^{n-1}$.

Theorem 5.5 (Qian [18]). Let $h=u+\omega v$ be radially differentiable in $\mathbb{R}^{n+1}$ and $n=2 k+1$. Then for any $k \in \mathbb{N}$ we find

$$
\tau_{n}(h)=\frac{1}{2^{k} k} \Delta^{k} h=(k-1) !\left[\left(\frac{1}{|\underline{x}|} \ell_{\omega}\right)^{k} u+\omega\left(\ell_{\omega} \frac{1}{|\underline{x}|}\right)^{k} v\right] .
$$

Corollary 5.6. Let $k=1$. A radially quaternionic regular function $h=u+\omega v$ fulfils the partial differential equation

$$
|\underline{x}|^{2} \Delta h-2|\underline{x}| \ell_{\omega} h+2 \operatorname{Vec} h=0 .
$$

Remark. A componentwise consideration of equation (5.2) leads for the scalar part to the Laplace-Beltrami equation in the hyperbolic metric and for the vector components to the Laplace-Beltrami equation to the eigenvalue -2 .

Now we will give a lot of examples which will emphasize how effectively Fueter's mapping $\tau_{n}$ is working. For abbreviation we write

$$
b_{k}(x)=\frac{\Gamma\left(\frac{1}{2}-k\right)}{\Gamma(2 k+1))}\left(\frac{2}{|\underline{x}|}\right)^{\frac{k-1}{2}} \frac{1}{k 2^{k}} .
$$


Example 5.7 (Exponential function). Let $n=2 k+1$ and $h(x)=e^{x} \cdot$ We obtain by applying Fueter's mapping

$$
\operatorname{EXP}_{k} x:=b_{k}(x)\left[J_{k-\frac{1}{2}}(|\underline{x}|)+\omega J_{k+\frac{1}{2}}(|\underline{x}|)\right] e^{x_{0}}
$$

where

$$
J_{\lambda}(|\underline{x}|)=\frac{\left(\frac{|\underline{x}|}{2}\right)^{\lambda}}{\Gamma\left(\frac{1}{2}\right) \Gamma\left(\frac{1}{2}+\lambda\right)} \int_{-1}^{1} e^{i|\underline{x}|^{t}}\left(1-t^{2}\right)^{\lambda-\frac{1}{2}} d t \quad(\lambda \in \mathbb{R})
$$

are Bessel functions of first kind. Indeed, we obtain from [10: p. 740] the equalities

$$
J_{k+\frac{1}{2}}(|\underline{x}|)=(-1)^{k+1} \sqrt{\frac{2}{\pi}}|\underline{x}|^{k+\frac{1}{2}}\left(\frac{1}{|\underline{x}|} \ell_{\omega}\right)^{k+1} \cos |\underline{x}|=(-1)^{k} \sqrt{\frac{2}{\pi}}\left(\ell_{\omega} \frac{1}{|\underline{x}|}\right)^{k} \sin |\underline{x}| .
$$

A straightforward calculation leads to the above defind exponential function $\operatorname{EXP}_{k} x$. In the special case for $k=1$ we have

$$
b_{1}(x)=-\frac{\Gamma\left(-\frac{1}{2}\right)}{2 !} \sqrt{\frac{2}{|\underline{x}|}}=\sqrt{\frac{2 \pi}{|\underline{x}|}}
$$

and

$$
\begin{aligned}
& J_{\frac{1}{2}}(|\underline{x}|)=\sqrt{\frac{2}{\pi|\underline{x}|}} \sin |\underline{x}| \\
& J_{\frac{3}{2}}(|\underline{x}|)=\sqrt{\frac{2}{\pi|\underline{x}|}}\left(\frac{\sin |\underline{x}|}{|\underline{x}|}-\cos |\underline{x}|\right) .
\end{aligned}
$$

Hence,

$$
\operatorname{EXP}_{1} x=e^{x_{0}}\left[\frac{\sin |\underline{x}|}{|\underline{x}|}+\omega\left(\frac{\sin |\underline{x}|-|\underline{x}| \cos |\underline{x}|}{|\underline{x}|^{2}}\right)\right]=e^{x_{0}}\left(\operatorname{sinc}|\underline{x}|-\omega \operatorname{sinc}^{\prime}|\underline{x}|\right)
$$

where $\operatorname{sinc}|\underline{x}|=\frac{\sin |\underline{x}|}{|\underline{x}|}$ and $\sin c^{\prime}|\underline{x}|:=\frac{d}{d|\underline{x}|}(\operatorname{sinc}|\underline{x}|)$.

In [21] F. Sommen obtained by using of a similarity principle for Vekua systems a class of hypercomplex exponential functions which can be seen as generalization of Fueter type mappings.

Example 5.8 (Hyperbolic functions). Let $n=2 k+1$. Further, let. $h(x)$ the radially hyperbolic sine function. We get

$$
\operatorname{SINH} x:=\tau_{n}(\sinh x)=b_{k}(x)\left[\sinh x_{0} J_{k-\frac{1}{2}}(|\underline{x}|)+\omega \cosh x_{0} J_{k+\frac{1}{2}}(|\underline{x}|)\right]
$$

and in the special case $k=1$ we have

$$
\operatorname{SINH} x:=\sinh x_{0} \operatorname{sinc}|\underline{x}|-\omega \cos x_{0} \sin \mathrm{c}^{\prime}|\underline{x}| \text {. }
$$


Let $h(x)$ the radially cosine function. We obtain

$$
\operatorname{COSH} x:=\tau_{n}(\cosh x)=b_{k}(x)\left[\cosh x_{0} J_{k-\frac{1}{2}}(|\underline{x}|)+\omega \sinh x_{0} J_{k+\frac{1}{2}}(|\underline{x}|)\right],
$$

and in the special case $k=1$ we have

$$
\operatorname{COSH} x:=\cosh x_{0} \operatorname{sinc}|\underline{x}|-\omega \sinh x_{0} \sin c^{\prime}|\underline{x}| .
$$

Example 5.9 (Trigonometric functions). Let $n=2 k+1$. It is easy to see that

$$
y=\omega(x) x=-|\underline{x}|+\frac{\underline{x} x_{0}}{|\underline{x}|} .
$$

Hence, $|\underline{y}|=\left|x_{0}\right|$ and $\omega(y)=\omega(x) \frac{x_{0}}{\left|x_{0}\right|}$. It follows that

$$
\operatorname{EXP}_{k} y=b_{k}\left(x_{0}\right)\left[J_{k-\frac{1}{2}}\left(|\underline{x}|_{0}\right)+\omega(x) \frac{x_{0}}{\left|x_{0}\right|} J_{k+\frac{1}{2}}\left(\left|x_{0}\right|\right)\right] e^{-\left|x_{0}\right|}
$$

We can now define a Clifford regular SIN-function by

$$
\begin{aligned}
\operatorname{SIN} x: & =\frac{1}{2}\left(\operatorname{EXP}_{k} y+\operatorname{EXP}_{k}(-y)\right) \omega(y) \\
& =b_{k}(x)\left[\cosh |\underline{x}| J_{k+\frac{1}{2}}\left(\left|x_{0}\right|\right)+\omega(x) \sinh |\underline{x}| J_{k-\frac{1}{2}}\left(\left|x_{0}\right|\right) \frac{x_{0}}{\left|x_{0}\right|}\right] .
\end{aligned}
$$

Analogously we can also define

$$
\begin{aligned}
\operatorname{COS} x: & =\frac{1}{2}\left[\operatorname{EXP}_{k} y+\operatorname{EXP}_{k}(-y)\right] \\
& =b_{k}\left(x_{0}\right)\left[\cosh |\underline{x}| J_{k-\frac{1}{2}}\left(\left|x_{0}\right|\right)-\omega(x) \sinh |\underline{x}| J_{k+\frac{1}{2}}\left(\left|x_{0}\right|\right) \frac{x_{0}}{\left|x_{0}\right|}\right] .
\end{aligned}
$$

In the special case $k=1$ we have

$$
\begin{aligned}
\operatorname{SIN} x & :=-\cosh |\underline{x}| \operatorname{sinc}^{\prime}\left|x_{0}\right|+\omega(x) \sinh |\underline{x}| \operatorname{sinc} x_{0} \frac{x_{0}}{\left|x_{0}\right|} \\
\operatorname{COS} x & :=\cosh |\underline{x}| \operatorname{sinc}\left|x_{0}\right|+\omega(x) \sinh |\underline{x}| \operatorname{sinc} c^{\prime}\left|x_{0}\right| \frac{x_{0}}{\left|x_{0}\right|} .
\end{aligned}
$$

Now we will give an application of this conception of elementary functions.

Theorem 5.10. Let $n=3$. The exponential function $\operatorname{EXP}_{3} x$ fulfils the property

$$
\bar{\partial} \operatorname{EXP}_{3}(\lambda x)=\lambda \operatorname{EXP}_{3} x \quad(\lambda \in \mathbb{C}) .
$$

Proof. Using the decomposition $D=|\underline{x}|^{-1} L+\omega \ell_{\omega}$ we obtain

$$
\begin{aligned}
\left(|\underline{x}|^{-1} L\right. & \left.+\omega \ell_{\omega}\right)\left[\frac{\sin \lambda|\underline{x}|}{\lambda|\underline{x}|}-\omega(x) \ell_{\omega}\left(\frac{\sin \lambda|\underline{x}|}{\lambda|\underline{x}|}\right)\right] \\
= & \frac{1}{\lambda}\left[|\underline{x}|^{-1} L\left(\frac{\sin \lambda|\underline{x}|}{|\underline{x}|}\right)+\omega \ell_{\omega}\left(\frac{\sin \lambda|\underline{x}|}{|\underline{x}|}\right)\right] \\
& -\left[\omega(x) \ell_{\omega} \omega(x) \ell_{\omega}\left(\frac{\sin \lambda|\underline{x}|}{|\underline{x}|}\right)-|\underline{x}|^{-1} L\left(\omega(x) \ell_{\omega} \frac{\sin \lambda|\underline{x}|}{|\underline{x}|}\right)\right] .
\end{aligned}
$$


From Proposition 4.2/(i) immediately

$$
L\left(\frac{\sin \lambda|\underline{x}|}{|\underline{x}|}\right)=0
$$

and

$$
\frac{1}{\lambda|\underline{x}|} L\left(\omega \ell_{\omega} \frac{\sin \lambda|\underline{x}|}{|\underline{x}|}\right)=-\frac{2}{\lambda|\underline{x}|} \ell_{\omega}\left(\frac{\sin \lambda|\underline{x}|}{|\underline{x}|}\right)=-\frac{2 \sin \lambda|\underline{x}|}{\lambda|\underline{x}|^{3}}+\frac{\cos \lambda|\underline{x}|}{|\underline{x}|^{2}}
$$

follows. It remains to consider

$$
\frac{1}{\lambda} \omega \ell_{\omega}\left(-\omega \ell_{\omega} \frac{\sin \lambda|\underline{x}|}{|\underline{x}|}\right)=\frac{1}{\lambda} \ell_{\omega}^{2}\left(\frac{\sin \lambda|\underline{x}|}{|\underline{x}|}\right) .
$$

A straightforward computation delivers

$$
\frac{1}{\lambda} \ell_{\omega}^{2}\left(\frac{\sin \lambda|\underline{x}|}{|\underline{x}|}\right)=\frac{\lambda \sin \lambda|\underline{x}|}{|\underline{x}|}-2 \frac{\cos \lambda|\underline{x}|}{|\underline{x}|^{2}}+\frac{2 \sin \lambda|\underline{x}|}{\lambda|\underline{x}|^{3}} .
$$

Finally, we have $D \operatorname{EXP}_{3}(\lambda x)=-\lambda \operatorname{EXP}_{3}(\lambda x)$ and so $\bar{\partial} \operatorname{EXP}_{3}(\lambda x)=\lambda \operatorname{EXP}_{3}(\lambda x)$

From the definition of the exponential function it follows now

Corollary 5.11. We have $\left|\operatorname{EXP}_{3} x\right|^{2}>0$ and $\lim _{\underline{x} \rightarrow 0} \operatorname{EXP}_{3} x=e^{x_{0}}$.

We consider now the so-called $\bar{\partial}$-problem and obtain the following result.

Corollary 5.12. Let $L_{n}=a_{n} \bar{\partial}^{n}+\ldots+a_{1} \bar{\partial}+a_{0} \quad\left(a_{k} \in \mathbb{R}\right)$. Further, let $\lambda_{k}$ be the roots of the algebraic equation $a_{n} \lambda^{n}+\ldots+a_{1} \lambda+a_{0}=0$. Then

$$
u_{k}=\operatorname{EXP}_{3} \lambda_{k} x \quad(k=1, \ldots, n)
$$

belong to $\operatorname{ker} L_{n}$, e.g. we have constructed a set of solutions of the $n$-th order linear partial differential equation $L_{n} u=0$ relatively to the operator $\bar{\partial}$.

Proof. We have to make the ansatz $u=\operatorname{EXP}_{3} \lambda x$. The result follows by using Theorem 5.10

\section{References}

[1] Brackx, F., Delanghe, R. and F. Sommen: Clifford Analysis. Boston - London - Melbourne: Pitman 1982.

[2] Common, A. K. and F. Sommen: Axial monogenic functions from holomorphic functions. J. Math. Anal. Appl. 179 (1993), $610-628$.

[3] De Graaf, J.: Note on the use of spherical vectorfields in Clifford analysis. In: Clifford Algebras and their Applications in Mathematical Physics (eds.: F. Brackx et al.). Dordrecht: Kluwer Acad. Publ. 1993, pp. $91-100$.

[4] Delanghe, R., Sommen, F. and V. Soucek: Clifford Algebra and Spinor-Valued Functions. Dordrecht: Kluwer Acad. Publ. 1992. 
[5] Fueter, R.: Analytische Theorie einer Quaternionenvariablen. Comment. Math. Helv. 4 (1932), $9-20$.

[6] Fueter, R.: Die Funktionentheorie der Differentialgleichungen $\Delta u=0$ und $\Delta \Delta u=0$ mit vier reellen Variablen. Comm. Math. Helv. 7 (1935), $307-330$.

[7] Fueter, R.: Reguläre Funktionen einer Quaternionenvariablen. Zürich: Universität 1949.

[8] Gürlebeck, K. and W. Sprössig: Quaternionic Calculus for Physicists and Engineers (Mathematical Methods in Practice: Vol. 1). Chichester: John Wiley \& Sons 1997.

[9] Imaeda, M.: On regular functions of a power-associative hypercomplex variable. In: Proc. Clifford Algebras and their Applications in Mathematical Physics, Canterbury 1985 (eds.: J. S. R. Chisholm and A. K. Common; D. Reidel NATO ASI Series C: Vol. 183). Dordrecht - Boston: Reidel 1986, pp. 565 - 572.

[10] Jank, G. and F. Sommen: Clifford analysis, biaxial symmetry and pseudoanalytic functions. Complex variables, Theory and Applications 13 (1990), $195-212$.

[11] Lawrentjew, M. A. and B. W. Schabat: Methoden der komplexen Funktionentheorie. Berlin: Dt. Verlag Wiss. 1965.

[12] Leutwiler, H.: Modified Clifford analysis. Complex Variables, Theory and Appl. 17 (1992), $153-171$.

[13] Leutwiler, H.: Modified quaternionic analysis in $\mathbb{R}^{3}$. Complex Variables 20 (1992), 19 51.

[14] Leutwiler, H.: More on modified quaternionic analysis in $\mathbb{R}^{3}$. Forum Math. 7 (1995), 279 $-305$.

[15] Lounesto, P. and P. Bergh: Axially symmetric vector fields and their complex potentials. Complex Variables, Theory and Appl. 2 (1983), 139 - 150.

[16] Marinov, M. S.: On the S-regular functions. J. Nat. Geom. 7 (1995), $21-44$.

[17] Nono, K.: Regularity of functions with values in Clifford algebras based on a generalized axially symmetric potential theory operator. In: Clifford Algebras and Their Applications in Mathematical Physics (Fundamental Theories of Physics: Vol. 55; eds.: F. Brackx et al.). Dordrecht: Kluwer Acad. Publ. 1993, pp. $159-166$.

[18] Qian, T.: Generalization of Fueter's result to $\mathbb{R}^{n+1}$. Rend. Mat. Acc. Lincei 8 (1997)9, $111-117$.

[19] Sce, M.: Osservazioni sulle serie di potenze nei moduli quadtratici. Atti Acc. Lincei Rend. fis. 23 (1957), $220-225$.

[20] Sommen, F.: A product and an exponential function in hypercomplex function theory. Appl. Anal. 12 (1981), $13-26$.

[21] Sommen, F.: Special functions in Clifford analysis and axial symmetry. .J. Math. Anal. Appl.: 130 (1988), $100-133$.

[22] Sudbery, A.: Quaternionic analysis. Proc. Camb. Phil. Soc. 85 (1979), $199-225$.:

[23] Van Acker, N.: Clifford differentiaaloperatoren en randwaardetheorie van de nuloplossingen ervan op de sfer en de Lie-sfer. Proefschrift. Gent: Rijksuniversiteit 1992. 